\title{
Do Herbig Ae/Be Stars Have Disks?
}

\author{
T.P. Ray and M. Corcoran \\ School of Cosmic Physics, Dublin Institute for Advanced Studies, 5 \\ Merrion Square, Dublin 2, Ireland
}

\begin{abstract}
.
The Herbig Ae/Be stars are optically visible pre-main sequence stars of intermediate mass $\left(\mathrm{M}_{*} \approx 3-8 \mathrm{M}_{\odot}\right)$ and are thought to be the higher mass analogues of the T Tauri stars. While there is no doubt that classical T Tauri stars, i.e. those with $\mathrm{EW}(\mathrm{H} \alpha) \gtrsim 10 \AA$, are surrounded by disks, it remains controversial as to whether this is the case with the equivalent Herbig Ae/Be stars. It has even been questioned whether the powerful winds that are ejected by Herbig Ae/Be stars are driven by accretion. To address these problems we have examined a large sample of these stars with the idea of using their forbidden line emission as an indirect diagnostic for the presence of disks. Striking similarities with the classical T Tauri stars are found. For example we have discovered evidence not only for a strong correlation between near-infrared colours and the equivalent width of the forbidden line emission but also that the forbidden line emission normally arises in a blueshifted outflow component. It has already been shown in the case of the classical T Tauri stars that the correlation of near-infrared colour with forbidden line equivalent width is due to a link between the accretion rate and the outflow rate. The virtually identical relationship seen in the case of the Herbig Ae/Be stars must then also have a similar origin. Our finding that the forbidden line emission in Herbig Ae/Be stars is normally blueshifted shows not only that it arises in an outflow but, as in the classical T Tauri stars, such an asymmetry in the velocity centre of the line must be caused by the obscuring effects of a disk. We find that the correlation seen in the classical $\mathrm{T}$ Tauri stars between the mass-loss rate and infrared excess can be extended, when we include the Herbig Ae/Be stars, to cover almost 5 orders of magnitude in stellar luminosity. Our observations therefore broaden the findings of earlier observers for low mass young stars and indicate the presence of circumstellar disks around the majority of Herbig Ae/Be stars with forbidden line emission. A corollary of our results is that the same outflow mechanism must operate in both the classical $\mathrm{T}$ Tauri stars and the Herbig $\mathrm{Ae} / \mathrm{Be}$ stars with forbidden line emission.
\end{abstract}

\section{Introduction}

Herbig (1960) was the first to identify a group of $\mathrm{H} \alpha$ emission line stars, now known as the Herbig Ae/Be stars (HAEBESs), as possible higher mass analogues 
of the T-Tauri stars (TTSs). Subsequent studies have shown that these two classes of object share many observational characteristics in common over and above what one might expect on the basis of observational selection effects alone. For example, many HAEBESs possess strong winds as indicated by their P-Cygni profiles (Finkenzeller \& Mundt 1984) and in some cases by their thermal radio emission (Skinner et al. 1993). Like the classical T Tauri stars (CTTSs), most are found to have infrared excesses (e.g. Hillenbrand et al. 1992) and a few are also known to have extended optical and molecular outflows (see, for example, Levreault 1988; Mundt \& Ray 1994). Given the intimate connection between disks and outflows in CTTSs (see, for example, Edwards et al. 1993) it is natural to ask whether or not the standard accretion disk model, as developed to explain many of the observational features of low mass young stars (e.g. Bertout et al. 1988), is applicable to a large number of the higher mass HAEBESs? A number of authors have investigated the question of whether accretion disks surround HAEBES and it is fair to say that there is considerable debate on this topic (for opposing views see Hillenbrand et al. 1992; Berrilli et al. 1992; Hartmann et al. 1993; Böhm \& Catala 1994; Ghandour et al. 1994). In order to address this problem, we have attempted to see whether we can take the analogy between HAEBESs and TTSs further concentrating, in particular, on properties which, in the case of the TTSs, clearly point towards the presence of disks. Before doing so, it is worth briefly recalling the evidence for accretion disks around CTTSs and then to compare it with what is known for the HAEBESs.

That there are disks around CTTSs can no longer be doubted: they are seen directly, for example, in a number of cases using the Hubble Space Telescope (e.g. McCaughrean \& O'Dell 1996; Stapelfeldt et al. these proceedings; McCaughrean, these proceedings; Ray et al. 1996). Comparable observations for HAEBES do not exist at present so one must resort to probing for disks by indirect means. In the case of the CTTSs, the presence of a disk, plus in some cases an additional dusty envelope, can be inferred from the spectral energy distribution of these stars (Basri \& Bertout 1993). Although HAEBE stars possess spectral energy distributions which can, at least in principle, be modeled by disks (Hillenbrand et al. 1992) such models have been called into question (Hartmann et al. 1993) as there may be difficulties reproducing the near-infrared emission given the inferred high accretion rates. We must therefore resort to looking at another disk and accretion probe which has been successfully exploited in the case of the TTSs i.e. forbidden line emission.

\section{A comparison of the forbidden line emission of $T$ Tauri stars and Herbig Ae/Be stars}

We have made a spectroscopic study of approximately 50 Herbig Ae/Be stars using a range of instruments. Details are given elsewhere (Corcoran \& Ray 1996; Corcoran \& Ray 1997) but it suffices here to say that approximately half of our sample show forbidden line emission. Let us start by recalling the evidence for a link between forbidden line emission and disk accretion in CTTSs. Firstly it is well known that CTTSs and weak-line T Tauri stars can be distinguished not only by the equivalent width of their $\mathrm{H} \alpha$ emission but by the presence and absence of forbidden line emission respectively. As the former, unlike the latter, 

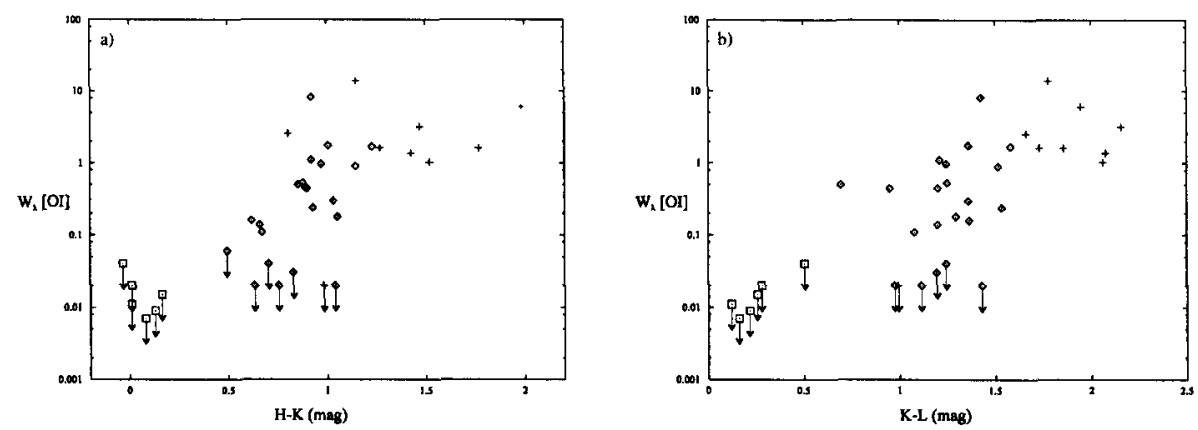

Figure 1. Plots of the relationship between EW([OI] $\lambda 6300)$ and H-K and K-L colour for the HAEBE stars. This is not our entire spectroscopic sample but only those for which good photometric data was available. For details, and an explanation of the symbols, see text.

are known to be surrounded by disks, this suggests a possible link between the presence of disks and forbidden line emission. In particular, for the CTTSs, see for example Edwards et al. (1993), it is found that the luminosity of the forbidden line emission scales with the near-infrared excess, i.e. the strength of the disk signature. Moreover, relative measures of the strength of the forbidden line emission, e.g. the equivalent width of the $[\mathrm{OI}] \lambda 6300$ line, scale with the nearinfrared colour in such a way that the redder sources tend to have the higher equivalent widths. Now one of the best measures we have of relative accretion, i.e. with respect to the underlying continuum of the star, is the "veiling" index. Veiling measures the degree to which absorption lines from the stellar photosphere are filled-in. Recently Hartigan et al. (1995) found a correlation between the degree of "veiling" and near-infrared colours in CTTSs. This implies not only a link between relative accretion and near-infrared colour but, by extension, a link between accretion and strength of the forbidden line emission. Is the same relationship between the equivalent width of the forbidden line emission and near-infrared colours seen in the case of the HAEBESs? If so then this could be taken as strong evidence not only that the same process, at least in general, is responsible for the forbidden line emission in both the CTTSs and HAEBESs but that this emission almost certainly arises as a result of accretion.

In Fig. 1 plots of the relationship between the equivalent width of the $[\mathrm{OI}] \lambda 6300$ line, $\mathrm{EW}([\mathrm{OI}] \lambda 6300)$, and $\mathrm{H}-\mathrm{K}$ and $\mathrm{K}-\mathrm{L}$ colour for the HAEBE stars are shown. There is a striking similarity between these plots and the corresponding ones in the case of the TTSs (Cabrit et al. 1990; Edwards et al. 1993) suggesting a common origin for the forbidden line emission. In addition, there are a number of features for the HAEBESs plots that are worth pointing out. In Fig. 1 a total of 3 types of Herbig Ae/Be star are included: the so-called Hillenbrand et al. (1992) Group III stars are shown as squares. These stars have no detectable forbidden line emission (hence the arrows indicating upper limits) and show only a small infrared excess. They may be the HAEBES analog to the weak-line TTSs. The others are either Hillenbrand Group I (diamond) or II (plus symbol) corresponding to stars with standard accretion or reprocessing 


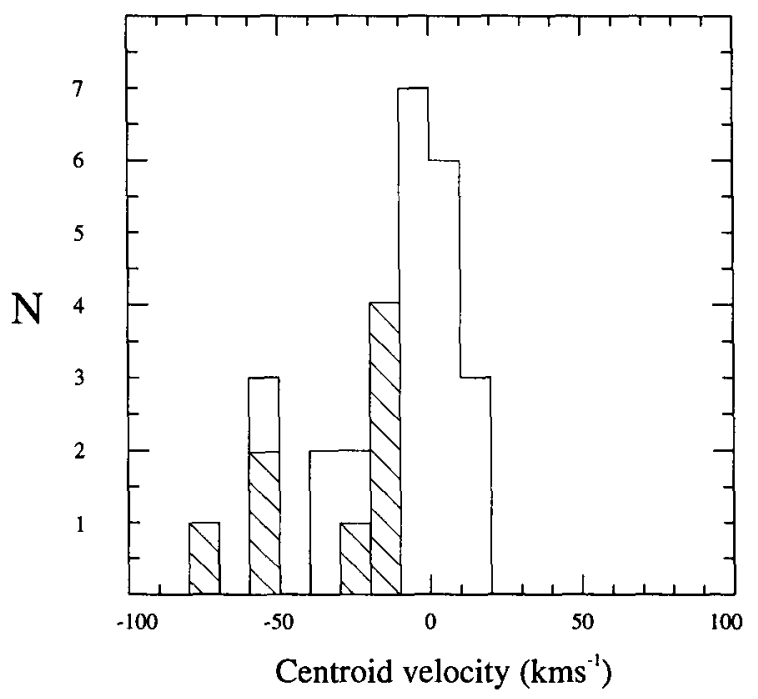

Figure 2. Histogram of the centroid velocities of the forbidden line emission for our sample of Herbig Ae/Be stars. Note the clear tendency for the emission to be blueshifted as expected if an occluding disk is present. Hillenbrand Group I and II stars (see text for an explanation) are unshaded and shaded respectively.

disk spectral energy distributions $\left(\lambda \mathrm{F}_{\lambda} \propto \lambda^{-4 / 3}\right)$ and those with a less steep drop in their infrared flux versus wavelength distribution respectively (Hillenbrand et al. 1992). The Group II stars are seen not only to be redder in H-K or K-L, as one would expect, but also to have stronger relative forbidden line emission. Given their higher degree of embeddedness, this suggests a link between evolutionary phase and relative strength of forbidden line emission. Searches for correlations between infrared excess and forbidden line luminosity, as a measure of mass loss rate, have also been made and it is found (Corcoran and Ray 1997) that the correlation seen in the case of the CTTSs between mass loss rate and infrared excess can be extended, when we include the Herbig $\mathrm{Ae} / \mathrm{Be}$ stars, to encompass 5 orders of magnitude in stellar luminosity.

When one examines the forbidden line emission of CTTSs, two components are often seen (Edwards, Ray and Mundt 1993): a high velocity component (with radial velocities typically in the range of $100-300 \mathrm{kms}^{-1}$ ) and a much lower velocity component (with radial velocities around $10 \mathrm{kms}^{-1}$ ). The high and low velocity components have been interpreted according to the model of Kwan and Tademaru (1988) as the jet and disk wind components respectively. For over a decade, the absence of high velocity redshifted forbidden lines in CTTSs (Appenzeller et al. 1984; Edwards et al. 1987) has been noted and is taken as evidence of opaque circumstellar disks: disks which occlude the receding component of the stellar wind or outflow, allowing only the blueshifted emission to be observed. Given the controversy that we have already alluded to as to whether a disk model is appropriate to Herbig Ae/Be stars, we have also undertook a 
search for such occluding effects in the forbidden line emission of our Herbig $\mathrm{Ae} / \mathrm{Be}$ star sample. It was found (Corcoran and Ray 1997) that those stars with forbidden [OI] $\lambda 6300$ emission (28 stars in total) can be divided into four distinct groups as determined by their line profiles and velocities. Roughly $15 \%$ (4 stars) of the sample show both high and low velocity blueshifted forbidden emission lines reminiscent of the line profiles of CTTSs with extended outflows. Of the three remaining groups, the first shows low velocity blueshifted emission with centroid velocities in the range $-55 \mathrm{kms}^{-1} \leq \mathrm{v}_{c} \leq-10 \mathrm{kms}^{-1}$ (14 stars), the second unshifted $\left(\left|v_{c}\right| \leq 5 \mathrm{kms}^{-1}\right)$ symmetrical forbidden emission lines $(7$ stars $)$ and the third group of 3 stars low velocity $\left(10 \mathrm{kms}^{-1} \leq \mathrm{v}_{c} \leq 15 \mathrm{kms}^{-1}\right)$ redshifted emission. No Herbig Ae/Be star was found to possess strongly redshifted forbidden line emission. The clear tendency towards blueshifted velocities (see Fig. 2) not only implicitly suggests the presence of occluding disks around these stars but there also appears to be a link between the degree of embeddedness and the amount of forbidden line shift. In particular the Hillenbrand Group II stars are seen to all have blueshifted low velocity emission, in addition to any high velocity component that might be present. An evolutionary effect may be responsible in the sense that, as the star becomes less enshrouded, the high velocity (jet) component of the forbidden line emission disappears first, followed by a decrease in the velocity of the low velocity component and finally by its disappearance altogether. The low velocity forbidden line emission is most likely a disk wind, the line profile being broadened as a result of the rotation of the disk. It is found that the line widths of the low velocity forbidden line emission are broader than those observed for the CTTSs. There is also evidence of acceleration in the outflow, at least in those Herbig Ae/Be stars with [SII] $\lambda \lambda 6717 / 6731$ emission in addition to the [OI] $\lambda 6300$ line. In particular when one compares the velocities of forbidden lines with higher and lower critical densities (i.e. [OI] $\lambda 6300$ and [SII] $\lambda \lambda 6717 / 6731$ respectively) it is found that the velocity increases as the critical density decreases suggestive of a wind leaving a disk (see Corcoran and Ray 1997).

\section{References}

Appenzeller, I., Jankovics, I., \& Östreicher, R., 1984, A\&A, 141, 108

Basri, G., \& Bertout, C., 1993, Protostars and Planets III, eds. Levy, E., Luine, J., University of Arizona Press p543

Berrilli, F., Corciulo, G., Ingrosso, G., Lorenzetti, D., Nisini, B., \& Strafella, F., 1992, ApJ, 398, 254

Bertout, C., Basri, G., \& Bouvier, J., 1988, ApJ, 330, 350

Böhm, T., \& Catala, C. 1994, A\&A, 290, 167

Cabrit, S., Edwards, S., Strom, S.E., \& Strom, K. M., 1990, ApJ, 354, 687

Corcoran, M., \& Ray, T.P., 1996, A\&A, submitted

Corcoran, M., \& Ray, T.P., 1996, A\&A, in press

Edwards, S.E., Cabrit, S., Strom, S.E., Heyer, I., Strom, K.M., \& Anderson, E., 1987, ApJ, 321, 473 
Edwards, S.E., Ray, T.P., \& Mundt, R., 1993, Protostars and Planets III, eds. Levy, E., Luine, J., University of Arizona Press p567

Finkenzeller, U., \& Mundt, R., 1984, A\&AS, 55, 109

Ghandour, L., Strom, S., Edwards, S., \& Hillenbrand, L., 1994, The Nature and Evolutionary Status of Herbig Ae/Be Stars, eds. Thé, P.S., Pérez, M.R., van den Heuvel, P.J., Astronomical Society of the Pacific Conference Series, p223

Hartigan, P., Edwards, S.E., \& Ghandour, L., 1995, ApJ, 452, 736

Hartmann, L., Kenyon, S.J., \& Calvet, N., 1993, ApJ, 407, 219

Hillenbrand, L.A., Strom, S.E., Vrba, F.J., \& Keene, J. 1992, ApJ, 397, 613

Herbig, G.H., 1960, ApJS, 4, 337

Kwan, J., \& Tademaru, E., 1988, ApJ, 322, L41

Leverault, R.M., 1988, ApJ, 330, 897

McCaughrean, M.J., \& O’Dell, C.R., 1996, AJ, 111, 1977

Mundt, R., \& Ray, T.P., 1994, The Nature and Evolutionary Status of Herbig Ae/Be Stars, eds. Thé, P.S., Pérez, M.R., van den Heuvel, P.J., Astronomical Society of the Pacific Conference Series, p237

Ray, T.P., Mundt, R., Dyson, J., Falle, S.A.E.G., \& Raga, A., 1996, ApJ, 468, L103

Skinner, S.L., Brown, A., \& Stewart, R.T., 1993, ApJS, 87, 217 